\title{
Data scientist
}

Daphne Ezer, Kirstie Whitaker

\section{Source}

Daphne Ezer, Kirstie Whitaker. (2019). Data science for the scientific life cycle.

Someone who develops new computational or statistical analysis techniques that can easily be adapted to a wide range of scenarios, or who can apply these techniques to answer a specific scientific question. 